\title{
A Strategy-Proof and Non-monetary Admission Control Mechanism for Wireless Access Networks
}

\author{
Xiaohan Kang, Juan José Jaramillo, Lei Ying \\ Department of Electrical and Computer Engineering \\ Iowa State University, Ames, IA, 50011 \\ Email: \{xkang,jjjarami,leiying $\} @$ iastate.edu
}

\begin{abstract}
We study admission control mechanisms for wireless access networks where (i) each user has a minimum service requirement, (ii) the capacity of the access network is limited, (iii) the access point is not allowed to use monetary mechanisms to guarantee that users do not lie when disclosing their minimum service requirements, and (iv) the access point wants to admit as many users as possible. To guarantee truthfulness, we use auction theory to design a mechanism where users compete to be admitted into the network. We propose admission control mechanisms under which the access point intelligently allocates resources based on the announced minimum service requirements to ensure that users have no incentive to lie and the capacity constraint is fulfilled. We also prove some properties that any feasible mechanism should have.
\end{abstract}

\section{Index Terms}

Auctions, truth-telling, admission control, resource allocation

\section{INTRODUCTION}

Resource allocation has been one of the most important issues in the design of communication networks. Given a wireless access network, in which the users have various quality of service (QoS) requirements and the access point has limited resources, admission control mechanisms are vital to achieve stability, fairness and efficiency of the system. In this paper, we consider a wireless access network with multiple users and a single access point. We assume that the access point network is a public network, and is not allowed to charge users for accessing the network. We study the case when the QoS requirements are private, and users are allowed to selfishly disclose any value that would give them better service. Then the problem is to design an admission control mechanism such that the true QoS of the users can be collected without the use of any pricing scheme, and as many users as possible are admitted. A natural choice is to set up a game with the users, such that the selfish users are incentivized to tell the truth. Originated in economics theory, auction mechanisms have been found very useful in this kind of situations, since they are designed to entice selfish bidders to tell the truth when allocating limited resources.

Various auction mechanisms have been well-studied. Myerson [2] has obtained the optimal auction mechanism in closed form mathematical expression. His result, however, only works for limited utility functions without constraints over the resource. The well-known VCG mechanism has been proved to guarantee truth-telling while achieving the social optimum [3]. The challenge of designing an effective auction mechanism in the proposed setting is the access point is not allowed to charge the users, so the auction mechanism has to be non-monetary. Thus, the VCG mechanism cannot be easily adapted in our scenario due to the non-monetary requirement. Credit schemes that were developed to incentivize cooperation in wireless networks could also be adapted to guarantee users do not lie about their true requirements, but they would also require secure mechanisms to avoid tampering with the virtual money [4], [5], [6], [7], [8], [9], [10]. Recently, Hou and Kumar have proposed a bidding game between users and

This paper is an extended version of an earlier paper that appeared in [1].

Research supported by NSF Grant CNS-0953165, and DTRA Grants HDTRA1-08-1-0016 and HDTRA1-09-1-0055. 
access point that maximizes the total utility, but in the iterative process the users are forced to bid specific values instead of bidding selfishly [11]. In [12] a knapsack auction is studied, where multiple bidders want to place objects of different sizes and valuations. While the problem also has capacity constraints, the object sizes are public knowledge and the auction is allowed to use payment schemes to guarantee users truthfully reveal their private valuations. A non-monetary mechanism is studied in [13], where only the user that requests the smaller service rate is admitted. This model is useful in peer-to-peer networks if the access technology does not provide separation between the upstream and downstream flows and users want to minimize the upload bandwidth in order to increase their download bandwidth. However, in the access point model that we study, the goal is to admit as many users as possible without violating the capacity constraint. In this paper, we seek to design auction mechanisms that are truthful and do not use any money-based scheme for general utility functions.

Our contributions are therefore threefold.

i. We model the admission control problem, whose objective is to admit as many users as possible, as an auction mechanism design with resource constraint.

ii. Second, we present two theorems that help us understand the essence of strategy-proof mechanisms. The first theorem (Theorem 2) shows the impossibility of a reasonable truth-telling mechanism to be based on probabilistic decisions, and the second theorem (Theorem 3) shows that the truthfulness of a mechanism is equivalent to the existence of a highest winning bid regardless of one's own bid.

iii. We propose our mechanism and show that it has the desired properties and admits at least half of the optimal number of users with high probability in an asymptotic sense.

This paper is organized as follows. Section $\amalg$ gives the model of this problem and the set of assumptions. Section 【II analyzes the problem and characterizes the properties for feasible auction mechanisms. Section IV gives our proposed mechanism and shows feasibility and the performance bound. Section $\nabla$ concludes this paper.

\section{MODEL}

Consider a multiple access network with $n$ users and a single access point (AP), where only one user can get a certain amount of resource allocated by the AP at any given time slot. Let $\mathcal{N}=\{1,2, \cdots, n\}$ be the set of all users. The AP is assumed to have resources with the total amount of $C>0$. Each user is assumed to have a quality of service (QoS) requirement $q_{i} \leq C$, which indicates the resource requested by the user $i$ and is only known to the user $i$. We suppose that the range of $q_{i}$ is $T_{i}=\left[a_{i}, b_{i}\right] \subset[0, C]$ and let $T=T_{1} \times T_{2} \times \cdots \times T_{n}$ and $T_{-i}=T_{1} \times T_{2} \times \cdots \times T_{i-1} \times T_{i+1} \times \cdots \times T_{n}$ where $\times$ denotes the Cartesian product. We use bold letters like $\mathbf{q}, \mathbf{t}, \mathbf{s} \in T$ and $\mathbf{q}_{-i}, \mathbf{t}_{-i}, \mathbf{s}_{-i} \in T_{-i}$ to imply vectors rather than scalars.

By setting $q_{i}^{\prime}=q_{i} / C$ and taking $q_{i}^{\prime}$ as the requested resource, the total amount of resources would then be 1 . So without loss of generality we set $C=1$ and call $q_{i}$ the service rate of user $i$ for the rest of the paper.

Our objective is to admit as many users as possible. Thus, in order to decide which users to serve and the QoS the AP should provide, the AP sets up an auction, in which each user, or bidder, bids a requested service rate $t_{i} \in T_{i}$, which can be different from the true service rate $q_{i}$, and the AP decides the set of users that get admitted and assigns service rate $x_{i}$ to user $i$. Note that the bid $\mathbf{t}$ is different from the bids in a traditional auction in that the higher a user bids, the more resource he/she is requesting, and thus the less likely he/she should be admitted. By Myerson's revelation principle [2], we only consider direct revelation mechanisms, i.e., the mechanisms in which users submit bids in the form of service rate values rather than in the form of any other strategies. Then the mechanism can be described by the outcome functions $(p, x)$, where $p$ and $x$ are the set of functions

$$
\begin{aligned}
& p(\mathbf{t})=\left(p_{\phi}(\mathbf{t}), \phi \subset \mathcal{N}\right) \\
& x(\mathbf{t})=\left(x_{i, \phi}(\mathbf{t}), \phi \subset \mathcal{N}\right)
\end{aligned}
$$


with $p_{\phi}: T \rightarrow \mathbb{R}$ being the probability that only the subset of users $\phi$ of $\mathcal{N}$ get admitted and $x_{i, \phi}: T \rightarrow \mathbb{R}$ being the actually assigned service rate for user $i$ if the subset $\phi$ is admitted. For convenience we set $x_{i, \phi}(\mathbf{t})$ to be the assigned service rate by mechanism $(p, x)$ if $i \in \phi$ and $p_{\phi}(t)>0$, and 0 otherwise, i.e., no resource is assigned to user $i$, which is equivalent to not admitting user $i$.

The utility function for user $i$, given the true service rate $q_{i}$ and the assigned service rate $x_{i}$, is $u_{i}\left(q_{i}, x_{i}\right)$. It is assumed that $u_{i}(\cdot, \cdot)$ is non-negative, non-decreasing with $x_{i}$ when $x_{i} \geq q_{i}$, and equals 0 when $x_{i}<q_{i}$. Given mechanism $(p, x)$, the expected utility for user $i$ with true service rate $q_{i}$, bid service rate $t_{i}$ and others bidding $\mathbf{t}_{-i}$ is

$$
U_{i}\left(p, x, q_{i}, t_{i}, \mathbf{t}_{-i}\right)=\sum_{\phi: i \in \phi} u_{i}\left(q_{i}, x_{i, \phi}(\mathbf{t})\right) p_{\phi}(\mathbf{t}) .
$$

We now present some definitions we will use throughout the paper.

Definition 1 (Incentive compatibility - IC). A mechanism $(p, x)$ is incentive compatible or truthful or strategy-proof if for any utility function, any $i \in \mathcal{N}$, any $\mathbf{q} \in T$ and any $t_{i} \in T_{i}$, we have

$$
U_{i}\left(p, x, q_{i}, q_{i}, \mathbf{q}_{-i}\right) \geq U_{i}\left(p, x, q_{i}, t_{i}, \mathbf{q}_{-i}\right)
$$

where $U(\cdot)$ is defined in (1). That is, any possible true service rate vector $\mathbf{q}$ is a Nash equilibrium [14], in which no user has incentive to lie if all the other users bid their true service rates.

It must be noted that the definition of IC requires no user has incentive to lie regardless of the utility function they have, as long as the assumptions on $u_{i}\left(q_{i}, x_{i}\right)$ hold. This concept of user utility is then fundamental to prove the results on truthfulness. However, it must be emphasized that our objective is to admit as many users as possible and not to maximize the total network utility.

Definition 2 (Weak-incentive compatibility - weak-IC). A mechanism $(p, x)$ is weakly-incentive compatible if for any utility function, for any $i \in \mathcal{N}$ and any $t_{i} \in T_{i}$, we have

$$
U_{i}\left(p, x, q_{i}, q_{i}, \mathbf{q}_{-i}\right) \geq U_{i}\left(p, x, q_{i}, t_{i}, \mathbf{q}_{-i}\right), \quad \forall \mathbf{q} \in T^{*}
$$

for some $T^{*} \subset T$ with $\mathcal{L}\left(T \backslash T^{*}\right)=0$, where $\mathcal{L}(\cdot)$ is the Lebesgue measure 1

Definition 3 (Feasibility). A mechanism $(p, x)$ is feasible if it satisfies

i. Probability constraint $(\mathrm{P})$ :

For any $\phi \subset \mathcal{N}$ and any $\mathbf{t} \in T$,

$$
\sum_{\psi \subset \mathcal{N}} p_{\psi}(\mathbf{t})=1 \quad \text { and } \quad p_{\phi}(\mathbf{t}) \geq 0
$$

ii. Capacity constraint (CC):

For any $i \in \mathcal{N}$, any $\mathbf{t} \in T$, and any $\phi \subset \mathcal{N}$ with $p_{\phi}(\mathbf{t})>0$,

$$
\sum_{j \in \phi} x_{j, \phi}(\mathbf{t}) \leq 1 \quad \text { and } \quad x_{i, \phi}(\mathbf{t}) \geq 0
$$

iii. Individual rationality (IR):

For any $\phi \subset \mathcal{N}$ with $p_{\phi}(\mathbf{t})>0$, any $\mathbf{t} \in T$, and any $i \in \phi$,

$$
x_{i, \phi}(\mathbf{t}) \geq t_{i} .
$$

iv. Incentive compatibility (IC) in (2).

\footnotetext{
${ }^{1}$ Lebesgue measure is the standard way to measure the subsets of an $n$-dimensional Euclidean space, which coincides with the standard measure of $n$-dimensional volumes.
} 
Definition 4 (Weak-determinism - weak-D). A mechanism $(p, x)$ is weakly-deterministic if for any $\mathbf{t} \in T$ and any $i \in \mathcal{N}$,

$$
\left(\forall j \in \mathcal{N}, j \neq i \Rightarrow t_{j} \neq t_{i}\right) \Rightarrow \sum_{\phi: i \in \phi} p_{\phi}(\mathbf{t}) \in\{0,1\} .
$$

That is, for a given bid vector $\mathbf{t}$, if user $i$ is the only one who bids $t_{i}$, then either the user always gets admitted, or the user never gets admitted.

Definition 5 (Determinism - D). A mechanism $(p, x)$ is deterministic if there exists a function $\psi: T \rightarrow$ $\mathcal{P}(\mathcal{N})$ such that for any $\mathbf{t} \in T$,

$$
p_{\phi}(\mathbf{t})= \begin{cases}1 & \text { if } \phi=\psi(\mathbf{t}) \\ 0 & \text { otherwise }\end{cases}
$$

where $\mathcal{P}(A)$ is the power set of $A$. Determinism implies that the winning set is always unique. We call $\psi(\cdot)$ the winning set function.

Note that for a deterministic mechanism $(p, x)$ we can have the assigned service rate denoted as

$$
x_{i}(\mathbf{t})=\left\{\begin{array}{ll}
x_{i, \psi(t)}(\mathbf{t}) & \text { if } i \in \psi(\mathbf{t}) \\
0 & \text { otherwise }
\end{array} .\right.
$$

Definition 6 (Anonymity). A mechanism $(p, x)$ is anonymous if for any $\mathbf{t} \in T$, any $\pi \in \Gamma_{n}$, and any $\phi \subset \mathcal{N}$,

$$
\begin{aligned}
p(\pi(\mathbf{t})) & =\pi(p(\mathbf{t})) \\
x_{\phi}(\pi(\mathbf{t})) & =\pi\left(x_{\phi}(\mathbf{t})\right),
\end{aligned}
$$

where $\Gamma_{n}$ is the set of all permutations of $n$ indices. That is, the outcome of the auction does not depend on the identity of the bidders.

Definition 7 (Monotonicity). A mechanism $(p, x)$ is monotonic if for any $i \in \mathcal{N}$, any $\mathbf{t}_{-i} \in T_{-i}$, and any $s_{i}, s_{i}^{\prime} \in T_{i}$ with $s_{i}>s_{i}^{\prime}$,

$$
\sum_{\phi: i \in \phi} p_{\phi}\left(\mathbf{t}_{-i}, s_{i}\right) \leq \sum_{\phi: i \in \phi} p_{\phi}\left(\mathbf{t}_{-i}, s_{i}^{\prime}\right) .
$$

That is, given that others' bids are fixed, a user's chance of getting admitted should not decrease when the user bids lower.

We have the following lemma for monotonicity.

Lemma 1. Any mechanism that satisfies $P, I R$ and IC is monotonic.

The proof of Lemma 1 is deferred to Appendix VI-A The idea of the proof is that if the mechanism is not monotonic, then there exists a utility function such that users have incentive to lie.

We are interested in feasible auction mechanisms that are weakly-deterministic and anonymous. Feasibility implies that the decision is in the capacity region, no user is forced to participate, and no one has incentive to lie about his type. Weak-determinism, anonymity and monotonicity are properties we consider desirable for a fair mechanism.

Definition 8 (Single-price). A mechanism $\mathcal{A}=(p, x)$ is single-priced if for any $\mathbf{t} \in T$ and any $\phi \subset \mathcal{N}$ with $p_{\phi}(\mathbf{t})>0$,

$$
x_{i, \phi}(\mathbf{t})=x_{j, \phi}(\mathbf{t}) \quad \forall i, j \in \phi .
$$

That is, all the users in a winning set get the same assigned service rate. 


\section{ANAlysis OF THE PROBlem}

In this section we analyze feasible strategy-proof mechanisms in non-monetary scenarios. We start with a mechanism $\overline{\mathcal{A}}=(\bar{p}, \bar{x})$, which is inspired by the uniform-price auction [3].

\section{A. The Simple Single-Priced Mechanism $\overline{\mathcal{A}}$}

We now propose the mechanism $\overline{\mathcal{A}}$, and prove that this mechanism satisfies the capacity constraint, is weak-IC, and admits at least half of the maximum possible number of users. After that we point out the flaws of the weak-IC concept.

Given bid vector $\mathbf{t} \in T$, the mechanism $\overline{\mathcal{A}}$ is described as follows.

Step i. Let $\alpha$ be a rearrangement of the indices such that $t_{\alpha(1)} \leq t_{\alpha(2)} \leq \cdots \leq t_{\alpha(n)}$. If several users bid the same, just arrange them randomly. Introduce a pseudo-bidder with $t_{n+1}=1$ and $\alpha(n+1)=n+1$.

Step ii. Find the largest index $\bar{m}$ with $1 \leq \bar{m} \leq n$ such that $\bar{m} \cdot t_{\alpha(\bar{m}+1)} \leq 1$. The winning set is $\bar{\psi}(t)=\{\alpha(1), \alpha(2), \cdots, \alpha(\bar{m})\}$.

Step iii. Set the assigned service rate $\bar{x}_{i}(\mathbf{t})=\bar{x}(\mathbf{t})=t_{\alpha(\bar{m}+1)}$ if $i \in \bar{\psi}(\mathbf{t})$.

The idea of the mechanism is basically that we start from the lower bidders and try to admit as many users as possible, with assigned service rate equal to the lowest losing bid.

Notice that the rearrangement $\alpha$ and the winning set $\bar{\psi}$ here might not be unique if several users bid the same value, in which case both of them will be probabilistic functions instead of deterministic functions. However, when there are no two users bidding the same value, $\alpha$ and $\bar{\psi}$ are deterministic functions.

Given the bids $\mathbf{t} \in T$ and a corresponding rearrangement $\alpha$, we let

$$
\sigma_{i}(\mathbf{t})=t_{\alpha(i)}, \quad 1 \leq i \leq n+1
$$

and

$$
\sigma(\mathbf{t})=\left(\sigma_{1}(\mathbf{t}), \sigma_{2}(\mathbf{t}), \cdots, \sigma_{n+1}(\mathbf{t})\right)
$$

where $\sigma(\mathbf{t})$ is the unique sorted vector of $\mathbf{t}$ with $\sigma_{1}(\mathbf{t}) \leq \sigma_{2}(\mathbf{t}) \leq \cdots \leq \sigma_{n+1}(\mathbf{t})$, regardless of the possible different choices of $\alpha$. Then the index chosen in step ii is a function of the bids given by

$$
\bar{m}(\mathbf{t})=\max \left\{m \in \mathcal{N} \mid m \cdot \sigma_{m+1}(\mathbf{t}) \leq 1\right\}
$$

where $\bar{m}(\mathbf{t})$ is always well-defined because

$$
1 \cdot t_{\alpha(2)} \leq 1
$$

Similarly, the assigned service rate for those admitted users is also determined by

$$
\bar{x}(\mathbf{t})=t_{\alpha(\bar{m}(\mathbf{t})+1)} .
$$

Example 1. Let the bid vector be $\mathbf{t}=\left(t_{1}, t_{2}, t_{3}, t_{4}\right)=\{0.5,0.4,0.3,0.4\}$.

Step $i$. The rearrangement could be $\alpha=(\alpha(1), \alpha(2), \alpha(3), \alpha(4), \alpha(5))=(3,2,4,1,5)$. ( $\alpha$ could also be $(3,4,2,1,5)$ ) So $t_{\alpha(1)} \leq t_{\alpha(2)} \leq t_{\alpha(3)} \leq t_{\alpha(4)} \leq t_{\alpha(5)}=1$ and the sorted vector of $\mathbf{t}$ is $\sigma(\mathbf{t})=(0.3,0.4,0.4,0.5,1)$.

Step ii. $2 \times 0.4=0.8 \leq 1$ and $3 \times 0.5=1.5>1$, so $\bar{m}(\mathbf{t})=2$ and the winning set is $\bar{\psi}(\mathbf{t})=\{2,3\}$ since $\alpha=(3,2,4,1,5)$. ( $\bar{\psi}(\mathbf{t})$ would be $\{3,4\}$ if $\alpha=(3,4,2,1,5)$.)

Step iii. The assigned service rate for either of the two winners is $\bar{x}(\mathbf{t})=t_{\alpha(3)}=t_{4}=0.4$. (Note that if $\alpha=(3,4,2,1,5)$, then $\bar{x}(\mathbf{t})$ would still be 0.4 .)

Lemma 2. The mechanism $\overline{\mathcal{A}}$ satisfies the $P, C C, I R$ and weak-IC constraints.

The proof of Lemma 2 is deferred to Appendix VI-B. It follows from directly checking the P, CC, and IR constraints. To verify weak-IC, we focus on the set of bid vectors with no equal bids from any two users. 
Note that $\overline{\mathcal{A}}$ does not satisfy feasibility because it is not IC. To see this, just consider two users bidding the same service rate. The chance of getting admitted is half for either user. The chance of either user getting admitted increases to 1 when he lower his bid by a small amount and the other user keeps the original bid. $\overline{\mathcal{A}}$, however, is weakly-IC. More specifically, for any $\mathrm{t}$ such that all bids are distinct, $\overline{\mathcal{A}}$ guarantees truth-telling.

We now show that $\overline{\mathcal{A}}$ admits at least half of the maximal possible number of users.

Theorem 1 (Scalability of $\overline{\mathcal{A}}$ ). For any true value of drop rate $\mathbf{t} \in T$, if there exists some mechanism with $P, C C$ and IR that admits $m$ users, then $\overline{\mathcal{A}}$ can admit at least $\left\lfloor\frac{m}{2}\right\rfloor$ users.

The proof of Theorem 1 is deferred to Appendix VI-C. The intuition behind it is that any algorithm that tries to admit more than $m$ users will violate $\mathrm{CC}$.

The problem about $\overline{\mathcal{A}}$ is that it is only weakly-IC but not IC. This means that if equal bids exist, users might have incentive to lie. For example, in a two-user case, if both users bid the same value, each of them would have half chance of getting admitted. But if one of them lower his bid by a small amount, he would win with the same assigned service rate and probability 1 . Thus indistinguishable bids make $\overline{\mathcal{A}}$ fail for IC.

\section{B. Impossibility for Probabilistic Decisions of Equal Bids}

We now show that to fulfill strict IC under some assumptions mentioned below, a weak-deterministic mechanism has to be deterministic. That is, if several users bid exactly the same value, then the only choice for guaranteeing truth-telling is to either admit all or none of them.

Theorem 2 (Impossibility). For a mechanism $\mathcal{A}$ that satisfies $P, I R, I C$ and anonymity, $\mathcal{A}$ is weaklydeterministic if and only if $\mathcal{A}$ is deterministic. That is, $\mathcal{A}$ admits either all or none of the equal bids.

The proof of Theorem 2 is deferred to Appendix VI-D, By definition we know that determinism implies weak-determinism, so we only need to prove that weak-determinism implies determinism. To do that, we prove by contradiction that if an auction is weakly-deterministic and not deterministic, then there exists utility functions such that users have an incentive to lie.

This theorem indicates that for any feasible and weakly-deterministic scheme, the ratio between the maximal possible number of users and the number of users admitted under the scheme is unbounded.

We should note that $\overline{\mathcal{A}}$ is weakly-deterministic because users might be randomly admitted when bidding the same. Then by Theorem 2, to achieve IC we need to design deterministic mechanisms, that is, mechanisms with only deterministic outcomes.

In the next section, we will present a feasible scheme based on $\overline{\mathcal{A}}$. Before that, we first present some properties any feasible scheme should have.

\section{Highest Winning Bid Theorem}

We further show that any deterministic mechanism with IC must be illustrated by a highest winning bid function.

Definition 9 (Highest winning bid mechanism). A deterministic mechanism $\mathcal{A}$ is a highest winning bid mechanism if there exists some function $z: T_{-i} \rightarrow T_{i}$ such that for any $\mathbf{t}_{-i} \in T_{-i}$,

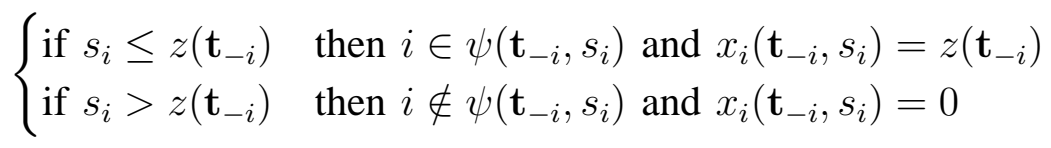

where $\psi(\cdot)$ is the winning set function of $\mathcal{A}$ defined in the definition of determinism (Definition 5).

The function $z(\cdot)$ is called the highest winning bid function of $\mathcal{A}$. 
Theorem 3 (Highest winning bid). A deterministic mechanism satisfies IC if and only if it is a highest winning bid mechanism.

The proof of Theorem 3 is deferred to Appendix VI-E] It can be checked that a highest winning mechanism satisfies IC, so we only need to prove the converse. To do that, we show that if a deterministic mechanism satisfies IC, then we can always construct a highest winning bid function.

The highest winning bid theorem shows us what a deterministic truth-telling mechanism should look like. Notice that no other assumptions are needed for this theorem, so it remains valid in a general setting. More importantly, this theorem gives us an efficient approach to design deterministic truth-telling mechanisms.

Although Theorem 3 does not work for $\overline{\mathcal{A}}$ due to weak-determinism, we do have the following similar result.

Lemma 3 (Supremum winning bid function for $\overline{\mathcal{A}}$ ). Under mechanism $\overline{\mathcal{A}}$, knowing others' bid $\mathbf{t}_{-i}$, the supremum of user i's winning bids is given by

$$
\bar{z}\left(\mathbf{t}_{-i}\right)=\max \left\{\sigma_{j}\left(\mathbf{t}_{-i}\right) \mid j \sigma_{j}\left(\mathbf{t}_{-i}\right) \leq 1\right\} .
$$

The proof of Lemma 3 is deferred to Appendix VI-F, It follows from checking that under $\overline{\mathcal{A}}$, and for all $i$, no bid larger than $\bar{z}\left(\mathbf{t}_{-i}\right)$ is admitted.

Note that $\bar{z}\left(\mathbf{t}_{-i}\right)$ is not the highest winning bid for user $i$ because bidding this value does not guarantee winning.

\section{Our Proposed Mechanism}

We now introduce the mechanism $\overline{\mathcal{A}}^{*}$, which is a truth-telling mechanism based on the previous $\overline{\mathcal{A}}$. We first construct $\overline{\mathcal{A}}^{*}$ by the so-called dropping trick. After that we show that $\overline{\mathcal{A}}^{*}$ is single-priced, feasible, and has very similar behavior to $\overline{\mathcal{A}}$.

\section{A. Dropping Trick}

The basic idea of the dropping trick is that since bidding exactly the supremum winning bid does not guarantee winning due to the capacity constraint, we drop the function by a small amount whenever necessary such that the capacity constraint is satisfied.

We would like to find a highest winning bid function $\bar{z}^{*}: T_{-i} \rightarrow T_{i}$ based on the supremum winning bid function $\bar{z}(\cdot)$ of $\overline{\mathcal{A}}$. For any $\mathbf{t}_{-i} \in T_{-i}$, let $\sigma_{0}\left(\mathbf{t}_{-i}\right)=0, \sigma_{n}\left(\mathbf{t}_{-i}\right)=1$, and let

$$
m_{1}\left(\mathbf{t}_{-i}\right)=\max _{j}\left\{j \mid j \sigma_{j}\left(\mathbf{t}_{-i}\right) \leq 1\right\}
$$

and

$$
m_{2}\left(\mathbf{t}_{-i}\right)=\max _{j}\left\{j \mid(j+1) \sigma_{j}\left(\mathbf{t}_{-i}\right) \leq 1\right\} .
$$

Then, we can also write the supremum winning bid function for $\overline{\mathcal{A}}$ as follows

$$
\bar{z}\left(\mathbf{t}_{-i}\right)=\sigma_{m_{1}\left(\mathbf{t}_{-i}\right)}\left(\mathbf{t}_{-i}\right) .
$$

It must be noted that from the definition of $m_{1}\left(\mathbf{t}_{-i}\right)$ and $m_{2}\left(\mathbf{t}_{-i}\right)$, whenever $m_{1}\left(\mathbf{t}_{-i}\right) \neq m_{2}\left(\mathbf{t}_{-i}\right)$, it must be the case that $m_{2}\left(\mathbf{t}_{-i}\right)<m_{1}\left(\mathbf{t}_{-i}\right)$. Thus, if $m_{1}\left(\mathbf{t}_{-i}\right) \neq m_{2}\left(\mathbf{t}_{-i}\right)$ we have from the definition of $m_{2}\left(\mathbf{t}_{-i}\right)$ that

$$
\left(m_{1}\left(\mathbf{t}_{-i}\right)+1\right) \sigma_{m_{1}\left(\mathbf{t}_{-i}\right)}\left(\mathbf{t}_{-i}\right)>1,
$$

or equivalently,

$$
\bar{z}\left(\mathbf{t}_{-i}\right)=\sigma_{m_{1}\left(\mathbf{t}_{-i}\right)}\left(\mathbf{t}_{-i}\right)>\frac{1}{m_{1}\left(\mathbf{t}_{-i}\right)+1} .
$$


For fixed parameters $\left(d_{j}, 1 \leq j \leq n-1\right)$ with $0<d_{j}<1$, the highest winning bid function of $\overline{\mathcal{A}}^{*}$ is defined as follows:

$$
\bar{z}^{*}\left(\mathbf{t}_{-i}\right)= \begin{cases}\bar{z}\left(\mathbf{t}_{-i}\right) & \text { if } m_{1}\left(\mathbf{t}_{-i}\right)=m_{2}\left(\mathbf{t}_{-i}\right) \\ \bar{z}\left(\mathbf{t}_{-i}\right)\left(1-d_{m_{1}\left(\mathbf{t}_{-i}\right)}\right)+\frac{1}{m_{1}\left(\mathbf{t}_{-i}\right)+1} d_{m_{1}\left(\mathbf{t}_{-i}\right)} & \text { if } m_{1}\left(\mathbf{t}_{-i}\right) \neq m_{2}\left(\mathbf{t}_{-i}\right)\end{cases}
$$

or equivalently

$$
\bar{z}^{*}\left(\mathbf{t}_{-i}\right)= \begin{cases}\sigma_{m_{1}\left(\mathbf{t}_{-i}\right)}\left(\mathbf{t}_{-i}\right) & \text { if } \sigma_{m_{1}\left(\mathbf{t}_{-i}\right)}\left(\mathbf{t}_{-i}\right) \leq \frac{1}{m_{1}\left(\mathbf{t}_{-i}\right)+1} \\ \sigma_{m_{1}\left(\mathbf{t}_{-i}\right)}\left(\mathbf{t}_{-i}\right)\left(1-d_{m_{1}\left(\mathbf{t}_{-i}\right)}\right)+\frac{1}{m_{1}\left(\mathbf{t}_{-i}\right)+1} d_{m_{1}\left(\mathbf{t}_{-i}\right)} & \text { if } \sigma_{m_{1}\left(\mathbf{t}_{-i}\right)}\left(\mathbf{t}_{-i}\right)>\frac{1}{m_{1}\left(\mathbf{t}_{-i}\right)+1}\end{cases}
$$

Thus, the parameter $d_{m_{1}\left(\mathbf{t}_{-i}\right)}$ drops the value of $\bar{z}^{*}\left(\mathbf{t}_{-i}\right)$ to lay on the interval $\left(\frac{1}{m_{1}\left(\mathbf{t}_{-i}\right)+1}, \bar{z}\left(\mathbf{t}_{-i}\right)\right)$ whenever $m_{1}\left(\mathbf{t}_{-i}\right) \neq m_{2}\left(\mathbf{t}_{-i}\right)$.

Note that, compared to the supremum winning bid function of $\overline{\mathcal{A}}$, we only do dropping when $m_{1}\left(\mathbf{t}_{-i}\right) \neq$ $m_{2}\left(\mathbf{t}_{-i}\right)$. We note that $\overline{\mathcal{A}}^{*}$ is a deterministic mechanism based on the highest winning bid function $\bar{z}^{*}(\cdot)$ in (9) and by Theorem 3 we know that $\overline{\mathcal{A}}^{*}$ satisfies IC.

We would like to highlight the fact that neither $\overline{\mathcal{A}}$ nor $\overline{\mathcal{A}}^{*}$ dominate the other in terms of maximizing the number of admitted users. That is, for some bid vectors $\overline{\mathcal{A}}$ admits more users than $\overline{\mathcal{A}}^{*}$ does and for some others $\overline{\mathcal{A}}^{*}$ admits more than $\overline{\mathcal{A}}$ does. For example, consider the 3 -user case and let the parameters for $\overline{\mathcal{A}}^{*}$ be $d_{1}=d_{2}=0.1$. For bid vector $(0.79,0.8,0.9)$, the first user would be admitted by $\overline{\mathcal{A}}$ with service rate 0.8 , but it would not be admitted by $\overline{\mathcal{A}}^{*}$ since $\bar{z}^{*}(0.8,0.9)=0.8-0.1 \times(0.8-0.5)=0.77<0.79$. Following a similar analysis for bid vector $(0.1,0.1,0.9)$, we note that $\overline{\mathcal{A}}$ only admits one user since it cannot admit two users with service rate 0.9 , while $\overline{\mathcal{A}}^{*}$ can admit two users since $\bar{z}^{*}(0.1,0.9)=0.1$.

Example 2. Take $d_{j}=0.1$ for any $1 \leq j \leq n-1$ in (9). Again, let the bid vector be $\mathbf{t}=\left(t_{1}, t_{2}, t_{3}, t_{4}\right)=$ $\{0.5,0.4,0.3,0.4\}$. By (8) we can calculate the supremum winning bid under $\overline{\mathcal{A}}$ for each user:

$$
\begin{aligned}
& \bar{z}\left(\mathbf{t}_{-1}\right)=\bar{z}(0.4,0.3,0.4)=0.4 \\
& \bar{z}\left(\mathbf{t}_{-2}\right)=\bar{z}(0.5,0.3,0.4)=0.4 \\
& \bar{z}\left(\mathbf{t}_{-3}\right)=\bar{z}(0.5,0.4,0.4)=0.4 \\
& \bar{z}\left(\mathbf{t}_{-4}\right)=\bar{z}(0.5,0.4,0.3)=0.4
\end{aligned}
$$

Then the highest winning bid under $\overline{\mathcal{A}}^{*}$ for each user is

$$
\begin{aligned}
& \bar{z}^{*}\left(\mathbf{t}_{-1}\right)=\bar{z}\left(\mathbf{t}_{-1}\right)-d_{2}\left(\bar{z}\left(\mathbf{t}_{-1}\right)-\frac{1}{3}\right) \doteq 0.3933 \\
& \bar{z}^{*}\left(\mathbf{t}_{-2}\right)=\bar{z}\left(\mathbf{t}_{-2}\right)-d_{2}\left(\bar{z}\left(\mathbf{t}_{-2}\right)-\frac{1}{3}\right) \doteq 0.3933 \\
& \bar{z}^{*}\left(\mathbf{t}_{-3}\right)=\bar{z}\left(\mathbf{t}_{-3}\right)-d_{2}\left(\bar{z}\left(\mathbf{t}_{-3}\right)-\frac{1}{3}\right) \doteq 0.3933 \\
& \bar{z}^{*}\left(\mathbf{t}_{-4}\right)=\bar{z}\left(\mathbf{t}_{-4}\right)-d_{2}\left(\bar{z}\left(\mathbf{t}_{-4}\right)-\frac{1}{3}\right) \doteq 0.3933 .
\end{aligned}
$$

Since only user 3's bid is lower than or equal to his highest winning bid, we have the winning set $\bar{\psi}^{*}(\mathbf{t})=\{3\}$ and $\bar{x}_{3}^{*}(\mathbf{t})=0.3933$.

\section{B. Properties of $\overline{\mathcal{A}}^{*}$}

We first notice that the dropping method above is chosen such that the mechanism remains single-priced.

Lemma 4. $\overline{\mathcal{A}}^{*}$ is single-priced.

The proof of Lemma 4 is deferred to Appendix VI-G. 
We then notice that the mechanism $\overline{\mathcal{A}}^{*}$ is indeed feasible.

Lemma 5. $\overline{\mathcal{A}}^{*}$ is feasible, and $0 \leq \bar{z}^{*}\left(\mathbf{t}_{-i}\right) \leq 1$ for any $\mathbf{t}_{-i} \in T_{-i}$.

The proof of Lemma 5 is deferred to Appendix VI-H. The key aspect of the proof is to show that the $\mathrm{CC}$ constraint is fulfilled. To do that, the proof considers the case when the dropping trick is used and when it is not. From that, and from the analysis of $\overline{\mathcal{A}}$, the capacity constraint can be verified.

\section{Performance Analysis}

We now have two single-priced mechanisms and would like to compare these to some optimal, nontruthful mechanisms that maximize the number of admitted users. We first introduce two optimal omniscient auctions [15].

Definition 10. Given bid vector $\mathbf{t}$, the optimal single price omniscient auction $\mathcal{F}$ admits the lowest $m_{\mathcal{F}}^{*}$ users with

$$
m_{\mathcal{F}}^{*}=\max \left\{m \mid m \sigma_{m}(\mathbf{t}) \leq 1\right\} .
$$

Compared to $\overline{\mathcal{A}}, \mathcal{F}$ use the highest winning bid as the universal price instead of the lowest losing bid. It must be noted though that $\mathcal{F}$ is non-deterministic and non-truthful.

Definition 11. Given bid vector $\mathrm{t}$, the optimal multiple price omniscient auction $\mathcal{T}$ admits the lowest $m_{\mathcal{T}}^{*}$ users with each winner's price equal to his own bid.

It is easy to see that $\mathcal{T}$ is not single-priced, non-deterministic and non-truthful, and admits the maximum number of users. To sum up, we list all the mechanisms we want to compare and their corresponding

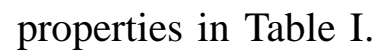

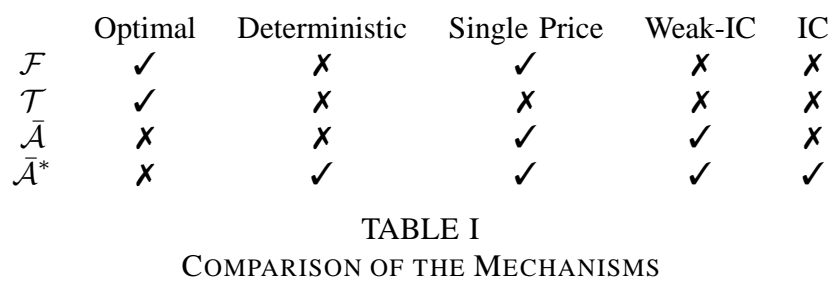

Definition 12. Given bid vector $\mathrm{t}$, the admittance of a mechanism $\mathcal{A}$ is the expected number of users admitted by $\mathcal{A}$, and we denote it by $|\mathcal{A}(\mathbf{t})|$.

We consider both Bayesian analysis and worst case analysis as follows.

1) Worst Case Analysis: First, by the scalability of $\overline{\mathcal{A}}$ we have

$$
|\overline{\mathcal{A}}(\mathbf{t})| \geq\left\lfloor\frac{|\mathcal{T}(\mathbf{t})|}{2}\right\rfloor
$$

for any $\mathbf{t} \in T$. This bound is tight since we can consider the bid vector with $m+1$ users bidding 0 and $m$ users bidding $\frac{1}{m}$, in which case $|\overline{\mathcal{A}}(\mathbf{t})|=m$ and $|\mathcal{T}(\mathbf{t})|=2 m+1$.

For single price mechanisms, we have the following worst case results.

Lemma 6. For any $\mathbf{t} \in T$,

$$
|\mathcal{F}(\mathbf{t})|-1 \leq|\overline{\mathcal{A}}(\mathbf{t})| \leq|\mathcal{F}(\mathbf{t})|
$$

The proof of Lemma 6 is deferred to Appendix VI-I, It follows from the definition of $\mathcal{F}(\mathbf{t})$ and $\overline{\mathcal{A}}(\mathbf{t})$. It is interesting to highlight that the bounds are tight since they are achievable. 
Lemma 7. For any $n \in \mathbb{N}$ and $m<n$, there exists some bid vector $\mathbf{t}$ such that

$$
\left|\overline{\mathcal{A}}^{*}(\mathbf{t})\right|=0, \quad|\mathcal{F}(\mathbf{t})| \geq m \text {. }
$$

That is to say, the worst case performance of $\overline{\mathcal{A}}^{*}$ could be arbitrarily bad.

The proof of Lemma 7 is deferred to Appendix VI-J] It uses the fact that in $\overline{\mathcal{A}}^{*}(\mathbf{t})$ all identical bids must be either accepted or rejected, while this is not the case for $\mathcal{F}(\mathbf{t})$. Note that while the worst case performance of $\overline{\mathcal{A}}^{*}$ can be bad, we will next show that the probability of getting a worst case can be made very small by setting small parameters for $\overline{\mathcal{A}}^{*}$.

2) Bayesian Analysis: We have shown that $\overline{\mathcal{A}}$ has roughly the same performance than $\mathcal{F}$ in terms of maximizing the number of admitted users.

Now we show that $\overline{\mathcal{A}}^{*}$ has very close performance to $\overline{\mathcal{A}}$. Assume that the drop rate vector $t$ is drawn from a distribution with joint probability density function $f: T \rightarrow \mathbb{R}^{+}$. Then, we have the following theorem.

Theorem 4. If the density function $f$ is upper-bounded by $K$, the probability that $\overline{\mathcal{A}}^{*}$ behaves differently from $\overline{\mathcal{A}}$ is at most $d n K$, where $d=\max _{1 \leq j \leq n-1} d_{j}$.

The proof of Theorem 4 is deferred to Appendix VI-K. It is based on the fact that if the density function is bounded, then the problem simplifies to bounding the Lebesgue measure of the set of bids where $\overline{\mathcal{A}}^{*}$ behaves differently from $\overline{\mathcal{A}}$.

We notice from Theorem 4 that the probability of different behaviors between the two mechanisms can be made arbitrarily small by choosing small parameters $\left(d_{j}, 1 \leq j \leq n-1\right)$.

\section{CONCLUSION}

In this paper, we studied the problem of designing a strategy-proof non-monetary auction mechanism for wireless networks. The motivation is to let the users tell the truth when bidding their resource requirements, and to admit as many users as possible. We gave a general model for the problem, analyzed the problem and found some properties that any strategy-proof auction mechanism should satisfy. Finally we proposed a feasible mechanism which is truthful even with equal bids, and showed that it could admit at least half of the maximum number of users with high probability in an asymptotic sense.

As possible topic for future work, discrete pricing models might be considered rather than continuous pricing models. Also, the assumption of weak-determinism could be weakened, and more specific utility functions could be considered for better performance. Furthermore, the lower bound of the number of admitted users might be improved.

\section{REFERENCES}

[1] X. Kang, J. J. Jaramillo, and L. Ying, "A strategy-proof and non-monetary admission control mechanism for wireless access networks," in Proc. 7th International ICST Conference on Heterogeneous Networking for Quality, Reliability, Security and Robustness (QShine), Houston, TX, USA, Nov. 17-19, 2010.

[2] R. B. Myerson, "Optimal auction design,” Mathematics of Operations Research, vol. 6, no. 1, pp. 58-73, Feb. 1981.

[3] V. Krishna, Auction Theory, 2nd ed. USA: Academic Press, 2010.

[4] L. Anderegg and S. Eidenbenz, "Ad hoc-VCG: A truthful and cost-efficient routing protocol for mobile ad hoc networks with selfish agents," in Proc. ACM MobiCom, San Diego, CA, Sep. 2003, pp. 245-259.

[5] L. Buttyán and J.-P. Hubaux, "Enforcing service availability in mobile ad-hoc WANs," in Proc. MobiHoc, Boston, MA, Aug. 2000, pp. 87-96.

[6] _ - "Stimulating cooperation in self-organizing mobile ad hoc networks," ACM/Kluwer Mobile Networks and Applications, vol. 8, no. 5, pp. 579-592, Oct. 2003.

[7] J. Crowcroft, R. Gibbens, F. Kelly, and S. Östring, "Modelling incentives for collaboration in mobile ad hoc networks," in Proc. WiOpt, France, Mar. 2003.

[8] V. Srinivasan, P. Nuggehalli, C. F. Chiasserini, and R. R. Rao, "Energy efficiency of ad hoc wireless networks with selfish users," in Proc. European Wireless Conference, Florence, Italy, Feb. 2002.

[9] —-, "Cooperation in wireless ad hoc networks," in Proc. IEEE INFOCOM, vol. 2, San Francisco, CA, Mar./Apr. 2003, pp. 808-817.

[10] S. Zhong, J. Chen, and Y. R. Yang, "Sprite: A simple, cheat-proof, credit-based system for mobile ad-hoc networks," in Proc. IEEE INFOCOM, vol. 3, San Francisco, CA, Mar./Apr. 2003, pp. 1987-1997. 
[11] I.-H. Hou and P. Kumar, "Utility maximization for delay constrained qos in wireless," in Proc. IEEE INFOCOM, San Diego, CA, Mar. 2010, pp. 1-9.

[12] G. Aggarwal and J. D. Hartline, "Knapsack auctions," in Proc. ACM-SIAM Symposium on Discrete Algorithms (SODA), Miami, FL, Jan. 22-26, 2006, pp. 1083-1092.

[13] M. Meo and F. Milan, "A rational model for service rate allocation in peer-to-peer networks," in Proc. IEEE INFOCOM, vol. 4, Miami, FL, Mar. 13-17, 2005, pp. 2798 - 2802.

[14] D. Fudenberg and J. Tirole, Game Theory. Cambridge, MA: The MIT Press, Aug. 1991.

[15] A. V. Goldberg, J. D. Hartline, A. R. Karlin, M. Saks, and A. Wright, "Competitive auctions," Games and Economic Behavior, vol. 55, no. 2, pp. 242-269, May 2006.

\section{APPENDIX}

\section{A. Proof of Lemma 1}

Proof: Let mechanism $(p, x)$ satisfy P, IR, IC. Suppose $(p, x)$ is not monotonic, then there exist $i \in \mathcal{N}, \mathbf{t}_{-i} \in T_{-i}$, and $s_{i}, s_{i}^{\prime} \in T_{i}$ such that $s_{i}>s_{i}^{\prime}$ and

$$
\sum_{\phi: i \in \phi} p_{\phi}\left(\mathbf{t}_{-i}, s_{i}\right)>\sum_{\phi: i \in \phi} p_{\phi}\left(\mathbf{t}_{-i}, s_{i}^{\prime}\right) .
$$

We consider the following utility function:

$$
u_{i}\left(t_{i}, x_{i}\right)=\left\{\begin{array}{ll}
d & \text { if } x_{i} \geq t_{i} \\
0 & \text { if } x_{i}<t_{i}
\end{array},\right.
$$

where $d$ is a positive constant. Let the true service rate value of user $i$ be $q_{i}=s_{i}^{\prime}$, then

$$
\begin{aligned}
U\left(p, x, q_{i}, s_{i}, \mathbf{t}_{-i}\right) & =U\left(p, x, s_{i}^{\prime}, s_{i}, \mathbf{t}_{-i}\right) \\
& =\sum_{\phi: i \in \phi} u_{i}\left(s_{i}^{\prime}, x_{i, \phi}\left(\mathbf{t}_{-i}, s_{i}\right)\right) p_{\phi}\left(\mathbf{t}_{-i}, s_{i}\right) \\
& =d \sum_{\phi: i \in \phi} p_{\phi}\left(\mathbf{t}_{-i}, s_{i}\right) \\
& >d \sum_{\phi: i \in \phi} p_{\phi}\left(\mathbf{t}_{-i}, s_{i}^{\prime}\right) \\
& =\sum_{\phi: i \in \phi} u_{i}\left(s_{i}^{\prime}, x_{i, \phi}\left(\mathbf{t}_{-i}, s_{i}^{\prime}\right)\right) p_{\phi}\left(\mathbf{t}_{-i}, s_{i}^{\prime}\right) \\
& =U\left(p, x, s_{i}^{\prime}, s_{i}^{\prime}, \mathbf{t}_{-i}\right) \\
& =U\left(p, x, t_{i}, t_{i}, \mathbf{t}_{-i}\right)
\end{aligned}
$$

where (11) comes from $x_{i, \phi}\left(\mathbf{t}_{-i}, s_{i}\right) \geq s_{i} \geq s_{i}^{\prime}$ when $p_{\phi}\left(\mathbf{t}_{-i}, s_{i}\right)>0$, (13) comes from $x_{i, \phi}\left(\mathbf{t}_{-i}, s_{i}^{\prime}\right) \geq s_{i}^{\prime}$ when $p_{\phi}\left(\mathbf{t}_{-i}, s_{i}^{\prime}\right)>0$, and (12) comes from (10). This contradicts IC. Thus, $(p, x)$ must be monotonic.

\section{B. Proof of Lemma 2}

Proof:

i. Probability constraint $(\mathrm{P})$ :

The probability constraint is obviously satisfied. For those $\mathbf{t}$ such that the winning set $\bar{\psi}(\mathbf{t})$ is determined, $\sum_{\phi \subset \mathcal{N}} \bar{p}_{\phi}(\mathbf{t})=\bar{p}_{\bar{\psi}(\mathbf{t})}(\mathbf{t})=1$. For those $\mathbf{t}$ such that there are $M$ possible winning sets, the probability for each of them would be $\frac{1}{M}$ and $\sum_{\phi \subset \mathcal{N}} \bar{p}_{\phi}(\mathbf{t})=M \cdot \frac{1}{M}=1$.

ii. Capacity constraint (CC): 
For any $\mathbf{t} \in T$ and any $\phi \subset \mathcal{N}$ with $\bar{p}_{\phi}(\mathbf{t})>0$,

$$
\begin{aligned}
\sum_{i \in \phi} \bar{x}_{i, \phi}(\mathbf{t}) & =\bar{m}(\mathbf{t}) \cdot \bar{x}(\mathbf{t}) \\
& =\bar{m}(\mathbf{t}) \cdot t_{\alpha(\bar{m}(\mathbf{t})+1)} \\
& \leq \bar{m}(\mathbf{t}) \cdot \frac{1}{\bar{m}(\mathbf{t})} \\
& =1 .
\end{aligned}
$$

Also $\bar{x}_{i, \phi}(\mathbf{t}) \geq 0$. Thus, $\overline{\mathcal{A}}$ satisfies CC.

iii. Individual rationality (IR):

For any $\mathbf{t} \in T$, any $\phi \subset \mathcal{N}$ with $\bar{p}_{\phi}(\mathbf{t})>0$, and any $i \in \phi$,

$$
\bar{x}_{i, \phi}(\mathbf{t})=\bar{x}(\mathbf{t})=t_{\alpha(\bar{m}(\mathbf{t})+1)} \geq t_{i} .
$$

iv. Weak-incentive compatibility (weak-IC):

We only consider the set of distinguishable bid vectors

$$
T_{D}=\left\{\mathbf{t} \in T \mid t_{i} \neq t_{j} \forall i \neq j\right\},
$$

that is, the set of bid vectors with no equal bids from any two users. For $\mathbf{t} \in T_{D}$ and $i \in \mathcal{N}$, the result of user $i$ bidding $s_{i}$ would be

$$
\bar{p}_{i}\left(\mathbf{t}_{-i}, s_{i}\right)= \begin{cases}1 & \text { if } s_{i}<\bar{x}(\mathbf{t}) \\ \frac{1}{2} & \text { if } s_{i}=\bar{x}(\mathbf{t}) \\ 0 & \text { if } s_{i}>\bar{x}(\mathbf{t})\end{cases}
$$

with assigned service rate $\bar{x}_{i}\left(\mathbf{t}_{-i}, s_{i}\right)=\bar{x}(\mathbf{t})$ if admitted. Note that $\bar{p}_{i}\left(\mathbf{t}_{-i}, s_{i}\right)=\frac{1}{2}$ when $s_{i}=\bar{x}(\mathbf{t})$ since there is only one other user who bids $\bar{x}(\mathbf{t})$.

We first consider the case of $i \in \bar{\psi}(\mathbf{t})$. We then have $t_{i}<\bar{x}(\mathbf{t})$. If $s_{i}<\bar{x}(\mathbf{t})$, then user $i$ still gets admitted with the same assigned service rate. If $s_{i}>\bar{x}(\mathbf{t})$, then user $i$ gets rejected. If $s_{i}=\bar{x}(\mathbf{t})$, then user $i$ either gets admitted with the same assigned service rate, or get rejected, both of which have probability $1 / 2$. So user $i$ cannot get better utility in the first case.

We then consider the case of $i \notin \bar{\psi}(\mathbf{t})$. Now we have $t_{i}>\bar{x}(\mathbf{t})$. If $s_{i}<\bar{x}(\mathbf{t})$, then user $i$ gets admitted with assigned service rate $\bar{x}(\mathbf{t})$ lower than true value $t_{i}$. If $s_{i}>\bar{x}(\mathbf{t})$, the user $i$ still does not get admitted. If $s_{i}=\bar{x}(\mathbf{t})$, then user $i$ either gets admitted with assigned service rate too low to accept, or does not get admitted at all, both of which have probability $1 / 2$. So user $i$ cannot get better utility in the second case.

Thus, for any $\mathbf{t} \in T_{D}$, no user has incentive to lie. As $T \backslash T_{D}$ has measure zero, we have weak-IC.

\section{Proof of Theorem $\square$}

Proof: Suppose $\overline{\mathcal{A}}$ admits only $m$ users, that is,

$$
\bar{m}(\mathbf{t})=m
$$

and some other mechanism $\mathcal{A}=(p, x)$ has a chance of admitting at least $2 m+2$ users given bid vector $\mathbf{t}$, that is,

$$
\exists \phi \subset \mathcal{N},|\phi| \geq 2 m+2, p_{\phi}(\mathbf{t})>0 .
$$


Then we have

$$
\begin{aligned}
\sum_{i \in \phi} x_{i, \phi}(\mathbf{t}) & \geq \sum_{i \in \phi} t_{i} \\
& \geq \sum_{i=1}^{2 m+2} \sigma_{i}(\mathbf{t}) \\
& \geq \sum_{i=m+2}^{2 m+2} \sigma_{i}(\mathbf{t}) \\
& \geq(m+1) \sigma_{m+2}(\mathbf{t}) \\
& >1
\end{aligned}
$$

where the inequality (14) comes from IR and (15) comes from the definition of the mechanism.

Thus $\mathcal{A}$ admits at most $2 m+1$ users. This is equivalent to the statement that if some mechanism with $\mathrm{P}, \mathrm{CC}$ and IR admits $m$ users, $\overline{\mathcal{A}}$ can at least admit $\left\lfloor\frac{m}{2}\right\rfloor$ users.

\section{Proof of Theorem 2}

Proof: We first prove the following lemma.

Lemma 8. A mechanism $\mathcal{A}=(p, x)$ is deterministic if and only if for any $\mathbf{t} \in T$ and any $i \in \mathcal{N}$,

$$
\sum_{\phi: i \in \phi} p_{\phi}(\mathbf{t}) \in\{0,1\}
$$

Proof: The determinism of $\mathcal{A}$ implies (16) because we have $\sum_{\phi: i \in \phi} p_{\phi}(\mathbf{t})=p_{\psi(t)}(\mathbf{t})=1$ for $i \in \psi(\mathbf{t})$, and $\sum_{\phi: i \in \phi} p_{\phi}(\mathbf{t})=0$ for $i \notin \psi(\mathbf{t})$.

If we have (16) for a mechanism $\mathcal{A}$, then we let

$$
\tilde{\psi}(\mathbf{t})=\left\{i \in \mathcal{N} \mid \sum_{\phi: i \in \phi} p_{\phi}(\mathbf{t})=1\right\}
$$

and claim that $\mathcal{A}$ is deterministic with the winning set function $\tilde{\psi}(\mathbf{t})$.

Indeed, suppose there exists $\psi \subset \mathcal{N}$ such that $p_{\psi}(\mathbf{t})>0$ and $\tilde{\psi}(\mathbf{t}) \neq \psi$. Then there exists $i \in$ $(\tilde{\psi}(\mathbf{t}) \backslash \psi) \cup(\psi \backslash \tilde{\psi}(\mathbf{t}))$. If $i \in \tilde{\psi}(\mathbf{t}) \backslash \psi$,

$$
\begin{aligned}
\sum_{\phi: i \in \phi} p_{\phi}(\mathbf{t}) & =\sum_{\substack{\phi: i \in \phi \\
\phi \neq \psi}} p_{\phi}(\mathbf{t}) \\
& \leq 1-p_{\psi}(\mathbf{t}) \\
& <1
\end{aligned}
$$

which contradicts the fact that $i \in \tilde{\psi}(\mathbf{t})$. If $i \in \psi \backslash \tilde{\psi}(\mathbf{t})$,

$$
\sum_{\phi: i \in \phi} p_{\phi}(\mathbf{t}) \geq p_{\psi}(\mathbf{t})>0
$$

which also contradicts the fact that $i \notin \tilde{\psi}(\mathbf{t})$. So for any $\psi, p_{\psi}(\mathbf{t})>0$ implies $p_{\psi}(\mathbf{t})=1$ and $\psi=\tilde{\psi}(\mathbf{t})$. Hence $\mathcal{A}$ is deterministic with winning set function $\tilde{\psi}(\mathbf{t})$.

By definition we readily have that determinism leads to weak-determinism.

On the other hand, suppose $\mathcal{A}$ is weakly-deterministic but not deterministic. By Lemma 8 , there exists $\mathbf{r} \in T$ and $i \in \mathcal{N}$ such that

$$
0<\sum_{\phi: i \in \phi} p_{\phi}(\mathbf{r})<1 .
$$


We first show that for any $r_{i}^{\prime}<r_{i}$, we have

$$
\sum_{\phi: i \in \phi} p_{\phi}\left(\mathbf{r}_{-i}, r_{i}^{\prime}\right)=1
$$

Indeed, let

$$
\tilde{r}_{i}=\left\{\begin{array}{ll}
\max _{j \in \mathcal{N}}\left\{r_{j} \mid r_{j}<r_{i}\right\} & \text { if } r_{j}<r_{i} \text { for some } j \in \mathcal{N} \\
0 & \text { otherwise }
\end{array} .\right.
$$

Then for any $r_{i}^{\prime} \in\left(\tilde{r}_{i}, r_{i}\right), r_{i}^{\prime}$ is a unique bid in $\left(\mathbf{r}_{-i}, r_{i}^{\prime}\right)$, so by monotonicity and weak-determinism we have $\sum_{\phi: i \in \phi} p_{\phi}\left(\mathbf{r}_{-i}, r_{i}^{\prime}\right)=1$. Again by monotonicity we also have $\sum_{\phi: i \in \phi} p_{\phi}\left(\mathbf{r}_{-i}, r_{i}^{\prime}\right)=1$ for $r_{i}^{\prime} \leq \tilde{r}_{i}$.

Let

$$
\Psi_{i}(\mathbf{r})=\left\{\phi \subset \mathcal{N} \mid i \in \phi, p_{\phi}(\mathbf{r})>0\right\} .
$$

Then $\Psi_{i}(\mathbf{r})$ is the set of possible winning sets that includes user $i$, given bid vector $\mathbf{r}$.

Now fix $r_{i}^{\prime}<r_{i}$, there are two cases. One is that there exists $\psi \in \Psi_{i}\left(\mathbf{r}_{-i}, r_{i}^{\prime}\right)$ with $x_{i, \psi}\left(\mathbf{r}_{-i}, r_{i}^{\prime}\right)<r_{i}$, and the other one is that for any $\psi \in \Psi_{i}\left(\mathbf{r}_{-i}, r_{i}^{\prime}\right), x_{i, \psi}\left(\mathbf{r}_{-i}, r_{i}^{\prime}\right) \geq r_{i}$. We want to prove that both cases lead to contradiction.

For the first case, there exists $\psi \in \Psi_{i}\left(\mathbf{r}_{-i}, r_{i}^{\prime}\right)$ with $x_{i, \psi}\left(\mathbf{r}_{-i}, r_{i}^{\prime}\right)<r_{i}$. Fix $r_{i}^{\prime \prime} \in\left(x_{i, \psi}\left(\mathbf{r}_{-i}, r_{i}^{\prime}\right), r_{i}\right)$. We consider the following utility function:

$$
u_{i}\left(t_{i}, x_{i}\right)= \begin{cases}u_{i}\left(x_{i}\right) & \text { if } x_{i} \geq t_{i} \\ 0 & \text { if } x_{i}<t_{i}\end{cases}
$$

with

$$
u_{i}\left(x_{i}\right)= \begin{cases}d & \text { if } x_{i} \geq r_{i}^{\prime \prime} \\ \frac{d}{r_{i}^{\prime \prime}} x_{i} & \text { if } x_{i}<r_{i}^{\prime \prime}\end{cases}
$$

where $d$ is a positive constant. Then for true values $\mathbf{t}_{-i}=\mathbf{r}_{-i}, t_{i}=r_{i}^{\prime}$ and possibly lying bid $s_{i}=r_{i}^{\prime \prime}$,

$$
\begin{aligned}
U_{i}\left(p, x, t_{i}, t_{i}, \mathbf{t}_{-i}\right)= & \sum_{\phi: i \in \phi} u_{i}\left(t_{i}, x_{i, \phi}\left(\mathbf{t}_{-i}, t_{i}\right)\right) p_{\phi}\left(\mathbf{t}_{-i}, t_{i}\right) \\
= & \sum_{\phi: i \in \phi, \phi \neq \psi} u_{i}\left(t_{i}, x_{i, \phi}\left(\mathbf{t}_{-i}, t_{i}\right)\right) p_{\phi}\left(\mathbf{t}_{-i}, t_{i}\right) \\
& +u_{i}\left(t_{i}, x_{i, \psi}\left(\mathbf{t}_{-i}, t_{i}\right)\right) p_{\psi}\left(\mathbf{t}_{-i}, t_{i}\right) \\
\leq & d \sum_{\phi: i \in \phi, \phi \neq \psi} p_{\phi}\left(\mathbf{t}_{-i}, t_{i}\right) \\
& +\left(\frac{d}{r_{i}^{\prime \prime}} x_{i, \psi}\left(\mathbf{r}_{-i}, r_{i}^{\prime}\right)\right) p_{\psi}\left(\mathbf{t}_{-i}, t_{i}\right) \\
< & d \sum_{\phi: i \in \phi, \phi \neq \psi} p_{\phi}\left(\mathbf{t}_{-i}, t_{i}\right)+d p_{\psi}\left(\mathbf{t}_{-i}, t_{i}\right) \\
= & d \sum_{\phi: i \in \phi} p_{\phi}\left(\mathbf{t}_{-i}, t_{i}\right) \\
= & d=U_{i}\left(p, x, t_{i}, s_{i}, \mathbf{t}_{-i}\right) .
\end{aligned}
$$

That is, user $i$ with true value $t_{i}$ has incentive to lie to bid $s_{i}$.

For the second case, for any $\psi \in \Psi_{i}\left(\mathbf{r}_{-i}, r_{i}^{\prime}\right)$, we have $x_{i, \psi}\left(\mathbf{r}_{-i}, r_{i}^{\prime}\right) \leq r_{i}$. We consider the following utility function:

$$
u_{i}\left(t_{i}, x_{i}\right)=\left\{\begin{array}{ll}
d & \text { if } x_{i} \geq t_{i} \\
0 & \text { if } x_{i}<t_{i}
\end{array},\right.
$$


where $d$ is a positive constant. Let the true values $\mathbf{t}_{-i}=\mathbf{r}_{-i}, t_{i}=r_{i}$, and the possible lying bid $s_{i}=r_{i}^{\prime}$. We have

$$
\begin{aligned}
U_{i}\left(p, x, t_{i}, t_{i}, \mathbf{t}_{-i}\right) & =d \sum_{\phi: i \in \phi} p_{\phi}(\mathbf{t}) \\
& <d=d \sum_{\phi: i \in \phi} p_{\phi}\left(\mathbf{t}_{-i}, s_{i}\right)=U_{i}\left(p, x, t_{i}, s_{i}, \mathbf{t}_{-i}\right) .
\end{aligned}
$$

That is, user $i$ with true value $t_{i}$ has incentive to lie to bid $s_{i}$. This contradiction completes the proof.

\section{E. Proof of Theorem 3}

Proof: Here we assume that a user always wins by the bid $s_{i}=0$, that is, for any $i \in \mathcal{N}$ and any $\mathbf{t}_{-i} \in T_{-i}$,

$$
\sum_{\phi: i \in \phi} p_{\phi}\left(0, \mathbf{t}_{-i}\right)=1 .
$$

Indeed, this is reasonable because by bidding a service rate of 0 the user is willing to drop all packets, thus the AP should always be able to admit this user. Also, $x_{i, \phi}\left(\mathbf{t}_{-i}, 0\right) \geq 0$ is true, so IR is always satisfied.

i. Suppose a deterministic mechanism $\mathcal{A}=(p, x)$ satisfies IC. Let the winning set function be $\psi(\cdot)$. We construct a function $z(\cdot)$ as follows. For any $\mathbf{t}_{-i} \in T_{-i}$, consider the winning bid set for user $i$

$$
W_{i}\left(\mathbf{t}_{-i}\right)=\left\{s_{i} \mid i \in \psi\left(\mathbf{t}_{-i}, s_{i}\right)\right\} .
$$

Then $W_{i}\left(\mathbf{t}_{-i}\right) \neq \emptyset$ since $0 \in W_{i}\left(\mathbf{t}_{-i}\right)$. We assert that for any $s_{i} \in W_{i}\left(\mathbf{t}_{-i}\right)$, the assigned service rate $x_{i}\left(\mathbf{t}_{-i}, s_{i}\right)$ is the same (recall that the assigned service rate for deterministic mechanisms can be denoted by single subscript, as in (7)). Indeed, if there exist $s_{i}, s_{i}^{\prime} \in W_{i}\left(\mathbf{t}_{-i}\right)$ with $x_{i}\left(\mathbf{t}_{-i}, s_{i}\right) \neq$ $x_{i}\left(\mathbf{t}_{-i}, s_{i}^{\prime}\right)$, we must have $s_{i} \neq s_{i}^{\prime}$. We may assume $x_{i}\left(\mathbf{t}_{-i}, s_{i}\right)>x_{i}\left(\mathbf{t}_{-i}, s_{i}^{\prime}\right)$. Let $t_{i}=s_{i}^{\prime}$, then given that other users bid $\mathbf{t}_{-i}$, user $i$ with true value $t_{i}$ has incentive to lie to bid $s_{i}$, since the assigned service rate would be higher. Thus the following function

$$
z\left(\mathbf{t}_{-i}\right)=\left\{x_{i}\left(\mathbf{t}_{-i}, s_{i}\right) \mid s_{i} \in W_{i}\left(\mathbf{t}_{-i}\right)\right\}
$$

is well defined.

Now we want to show that $z(\cdot)$ is the highest winning bid function of $(p, x)$. This is equivalent to showing that $W_{i}\left(\mathbf{t}_{-i}\right)=\left[0, z\left(\mathbf{t}_{-i}\right)\right]$. For any $s_{i} \leq z\left(\mathbf{t}_{-i}\right)$, if $s_{i} \notin W_{i}\left(\mathbf{t}_{-i}\right)$, then the user with true value $t_{i}=s_{i}$ has incentive to bid any winning bid $s_{i}^{\prime} \in W_{i}\left(\mathbf{t}_{-i}\right)$ since the assigned service rate $z\left(\mathbf{t}_{-i}\right) \geq t_{i}$, which contradicts IC. For any $s_{i}>z\left(\mathbf{t}_{-i}\right)$, if $s_{i} \in W_{i}\left(\mathbf{t}_{-i}\right)$, then the assigned service rate is $x_{i}\left(\mathbf{t}_{-i}, s_{i}\right)=z\left(\mathbf{t}_{-i}\right)<s_{i}$, which violates IR. Thus $W_{i}\left(\mathbf{t}_{-i}\right)=\left[0, z\left(\mathbf{t}_{-i}\right)\right]$, that is, $\mathcal{A}$ is a highest winning bid mechanism with highest winning bid function $z(\cdot)$.

ii. Suppose $\mathcal{A}$ is a highest winning bid mechanism with highest winning bid function $z: T_{-i} \rightarrow T_{i}$. Then when other users' bids $\mathbf{t}_{-i}$ are fixed, user $i$ with true value $t_{i} \leq z\left(\mathbf{t}_{-i}\right)$ has no incentive to lie, because bidding $s_{i} \leq z\left(\mathbf{t}_{-i}\right)$ results in the same assigned service rate and bidding $s_{i}>z\left(\mathbf{t}_{-i}\right)$ kicks user $i$ out. Meanwhile, user $i$ with true value $t_{i}>z\left(\mathbf{t}_{-i}\right)$ does not have incentive to lie either, since bidding $s_{i} \leq z\left(\mathbf{t}_{-i}\right)$ results in a assigned service rate too low to accept and bidding $s_{i}>z\left(\mathbf{t}_{-i}\right)$ keeps user $i$ out of admittance. Thus $\mathcal{A}$ satisfies IC.

\section{F. Proof of Lemma 3}

Proof: This can be checked by directly going through the process of $\overline{\mathcal{A}}$. If $t_{i}<\bar{z}\left(\mathbf{t}_{-i}\right)$, we can see that $\bar{x}(\mathbf{t})=\bar{z}\left(\mathbf{t}_{-i}\right)>t_{i}$, so user $i$ wins. If $t_{i}>\bar{z}\left(\mathbf{t}_{-i}\right)$, we have $\bar{x}(\mathbf{t}) \leq t_{i}$, so user $i$ loses. If $t_{i}=\bar{z}\left(\mathbf{t}_{-i}\right)$, user $i$ wins with some probability between 0 and 1 , which depends on the number of users bidding $\bar{z}\left(\mathbf{t}_{-i}\right)$. 


\section{G. Proof of Lemma 4}

Proof: By (9), we can see that the highest winning bid function of $\overline{\mathcal{A}}^{*}$ is actually a function of $\sigma_{m_{1}\left(\mathbf{t}_{-i}\right)}\left(\mathbf{t}_{-i}\right)$ and its ranking $m_{1}\left(\mathbf{t}_{-i}\right)$, that is,

$$
\bar{z}^{*}\left(\mathbf{t}_{-i}\right)=g\left(\sigma_{m_{1}\left(\mathbf{t}_{-i}\right)}\left(\mathbf{t}_{-i}\right), m_{1}\left(\mathbf{t}_{-i}\right)\right)
$$

so the highest winning bid function is determined once the $m_{1}\left(\mathbf{t}_{-i}\right)$ 'th highest bid and its ranking is determined.

Suppose $\overline{\mathcal{A}}^{*}$ is not single-priced, then there exists $\mathbf{t} \in T$ and $i, j \in \mathcal{N}$ such that

$$
t_{i} \leq \bar{z}^{*}\left(\mathbf{t}_{-i}\right), t_{j} \leq \bar{z}^{*}\left(\mathbf{t}_{-j}\right), \bar{z}^{*}\left(\mathbf{t}_{-i}\right) \neq \bar{z}^{*}\left(\mathbf{t}_{-j}\right) .
$$

Given $\tilde{\mathbf{t}} \in \prod_{k \neq i, k \neq j} T_{k}$, let

$$
f(t)=\bar{z}^{*}(\tilde{\mathbf{t}}, t)
$$

then we first claim that for any $\tilde{\mathbf{t}}$, there exists some $t_{0} \in[0,1]$ such that

$$
\left\{\begin{array}{ll}
f(t) \leq t & \text { for } t>t_{0} \\
f(t)=t_{0} & \text { for } t \leq t_{0}
\end{array} .\right.
$$

First, consider the case $f(0)=0$. Then for any $t>0$, if $f(t)>t$, then there exists $k \in\{1,2, \ldots, n-1\}$ such that

$$
\left\{\begin{array}{l}
\sigma_{k}(\tilde{\mathbf{t}}, t)=\bar{z}(\tilde{\mathbf{t}}, t) \geq \bar{z}^{*}(\tilde{\mathbf{t}}, t)=f(t) \\
k \sigma_{k}(\tilde{\mathbf{t}}, t) \leq 1 \\
(k+1) \sigma_{k+1}(\tilde{\mathbf{t}}, t)>1
\end{array},\right.
$$

which implies, by changing $t$ to 0 , we have

$$
\left\{\begin{array}{l}
\sigma_{k}(\tilde{\mathbf{t}}, 0)=\sigma_{k}(\tilde{\mathbf{t}}, t) \\
k \sigma_{k}(\tilde{\mathbf{t}}, 0)=k \sigma_{k}(\tilde{\mathbf{t}}, t) \leq 1 \\
(k+1) \sigma_{k+1}(\tilde{\mathbf{t}}, 0)=(k+1) \sigma_{k+1}(\tilde{\mathbf{t}}, t)>1
\end{array},\right.
$$

that is,

$$
m_{1}(\tilde{\mathbf{t}}, 0)=m_{2}(\tilde{\mathbf{t}}, t) \quad \text { and } \quad \sigma_{m_{1}(\tilde{\mathbf{t}}, 0)}(\tilde{\mathbf{t}}, 0)=\sigma_{m_{1}(\tilde{\mathbf{t}}, t)}(\tilde{\mathbf{t}}, t)
$$

and then by (19) we have

$$
f(t)=f(0)=0<t,
$$

which contradicts the assumption that $f(t)>t$. So $f(t) \leq t$ for any $t>0$. Thus we have $t_{0}=0$ in (21).

Now we consider the case $f(0)>0$. Then for any $t>f(0)$, if $f(t)>t$, then there exists $k \in$ $\{1,2, \ldots, n-1\}$ such that

$$
\left\{\begin{array}{l}
\sigma_{k}(\tilde{\mathbf{t}}, t)=\bar{z}(\tilde{\mathbf{t}}, t) \geq \bar{z}^{*}(\tilde{\mathbf{t}}, t)=f(t) \\
k \sigma_{k}(\tilde{\mathbf{t}}, t) \leq 1 \\
(k+1) \sigma_{k+1}(\tilde{\mathbf{t}}, t)>1
\end{array},\right.
$$

which implies, by changing $t$ to 0 , we again have

$$
\left\{\begin{array}{l}
\sigma_{k}(\tilde{\mathbf{t}}, 0)=\sigma_{k}(\tilde{\mathbf{t}}, t) \\
k \sigma_{k}(\tilde{\mathbf{t}}, 0)=k \sigma_{k}(\tilde{\mathbf{t}}, t) \leq 1 \\
(k+1) \sigma_{k+1}(\tilde{\mathbf{t}}, 0)=(k+1) \sigma_{k+1}(\tilde{\mathbf{t}}, t)>1
\end{array}\right.
$$

that is,

$$
m_{1}(\tilde{\mathbf{t}}, 0)=m_{2}(\tilde{\mathbf{t}}, t) \quad \text { and } \quad \sigma_{m_{1}(\tilde{\mathbf{t}}, 0)}(\tilde{\mathbf{t}}, 0)=\sigma_{m_{1}(\tilde{\mathbf{t}}, t)}(\tilde{\mathbf{t}}, t)
$$


and again by (19) we have

$$
f(t)=f(0)<t,
$$

which contradicts the assumption that $f(t)>t$. So $f(t) \leq t$ for any $t>f(0)$.

For $t^{\prime} \leq f(0)$, since $f(0)=\sigma_{m_{1}(\tilde{\mathbf{t}}, 0)}(\tilde{\mathbf{t}}, 0)$, we have

$$
m_{1}\left(\tilde{\mathbf{t}}, t^{\prime}\right)=m_{1}(\tilde{\mathbf{t}}, 0) \quad \text { and } \quad \sigma_{m_{1}\left(\tilde{\mathbf{t}}, t^{\prime}\right)}\left(\tilde{\mathbf{t}}, t^{\prime}\right)=\sigma_{m_{1}(\tilde{\mathbf{t}}, 0)}(\tilde{\mathbf{t}}, 0) .
$$

Then by (19), $f\left(t^{\prime}\right)=f(0)$ and thus we set $t_{0}=f(0)$ in (21). Therefore the claim is proved.

We then show that $\overline{\mathcal{A}}^{*}$ is single-priced. For any bid vector $\mathbf{t}$, let $\tilde{\mathbf{t}}$ be the bids in $\mathbf{t}$ other than $t_{i}$ and $t_{j}$, and let $t_{0}$ be such that (21) holds for $\tilde{\mathbf{t}}$, then by (20)

$$
t_{i} \leq \bar{z}^{*}\left(\mathbf{t}_{-i}\right)=\bar{z}^{*}\left(\tilde{\mathbf{t}}, t_{j}\right)=f\left(t_{j}\right)
$$

and

$$
t_{j} \leq \bar{z}^{*}\left(\mathbf{t}_{-j}\right)=\bar{z}^{*}\left(\tilde{\mathbf{t}}, t_{i}\right)=f\left(t_{i}\right) .
$$

First consider the case if $t_{i}>t_{0}$ and $t_{j}>t_{0}$. We have by (21)

$$
t_{i} \geq f\left(t_{i}\right) \geq t_{j} \geq f\left(t_{j}\right) \geq t_{i}
$$

and then $t_{i}=t_{j}$ and $\bar{z}^{*}\left(\mathbf{t}_{-i}\right)=f\left(t_{j}\right)=f\left(t_{i}\right)=\bar{z}^{*}\left(\mathbf{t}_{-j}\right)$.

Then consider the case if $t_{i}>t_{0}$ and $t_{j} \leq t_{0}$. Then by (21),

$$
t_{0}=f\left(t_{j}\right) \geq t_{i}
$$

which contradicts $t_{0}<t_{i}$, so this case is not possible.

Then consider the last case if $t_{i} \leq t_{0}$ and $t_{j} \leq t_{0}$. By (21),

$$
\bar{z}^{*}\left(\mathbf{t}_{-i}\right)=f\left(t_{j}\right)=t_{0}=f\left(t_{i}\right)=\bar{z}^{*}\left(\mathbf{t}_{-j}\right) .
$$

Thus we always have $\bar{z}^{*}\left(\tilde{\mathbf{t}}, t_{i}\right)=\bar{z}^{*}\left(\tilde{\mathbf{t}}, t_{j}\right)$, which contradicts the previous assumption. Therefore, $\overline{\mathcal{A}}^{*}$ is single-priced.

\section{H. Proof of Lemma 5}

Proof: P comes from determinism. IR comes from the definition of highest winning bid mechanisms. IC comes from Theorem 3. So only the proof of CC requires some more effort.

For given $\mathbf{t}$, we divide the winning users into two parts: those whose price is dropped, denoted by $A$ and those whose price is not dropped, denoted by $B$. Then

$$
\begin{aligned}
& A=\left\{i \in \bar{\psi}^{*}(\mathbf{t}) \mid \bar{z}^{*}\left(\mathbf{t}_{-i}\right)=\bar{z}\left(\mathbf{t}_{-i}\right)\right\} \\
& B=\left\{i \in \bar{\psi}^{*}(\mathbf{t}) \mid \bar{z}^{*}\left(\mathbf{t}_{-i}\right)<\bar{z}\left(\mathbf{t}_{-i}\right)\right\}
\end{aligned}
$$

where $\bar{\psi}^{*}(\cdot)$ is the winning set function for $\overline{\mathcal{A}}^{*}$. Then $A \cap B=\emptyset$ and $A \cup B=\bar{\psi}^{*}(\mathbf{t})$.

If $B=\emptyset$, then for any $i \in \bar{\psi}^{*}(\mathbf{t})$, let $m=m_{1}\left(\mathbf{t}_{-i}\right)$ and we have

$$
\begin{gathered}
(m+1) \sigma_{m+1}\left(\mathbf{t}_{-i}\right)>1 \\
(m+1) \sigma_{m}\left(\mathbf{t}_{-i}\right) \leq 1
\end{gathered}
$$

then

$$
\sigma_{m+1}\left(\mathbf{t}_{-i}\right)>\sigma_{m}\left(\mathbf{t}_{-i}\right)=\bar{z}\left(\mathbf{t}_{-i}\right)=\bar{z}^{*}\left(\mathbf{t}_{-i}\right)
$$

and

$$
\left|\bar{\psi}^{*}(\mathbf{t})\right| \leq m+1
$$


Then we have

$$
\begin{aligned}
\sum_{j \in \bar{\psi}^{*}(\mathbf{t})} \bar{x}_{j}^{*}(\mathbf{t}) & =\left|\bar{\psi}^{*}(\mathbf{t})\right| \cdot \bar{z}^{*}\left(\mathbf{t}_{-i}\right) \\
& \leq(m+1) \sigma_{m}\left(\mathbf{t}_{-i}\right) \\
& \leq 1
\end{aligned}
$$

where (22) follows because $\overline{\mathcal{A}}^{*}$ is single-priced and the other two inequalities come from the analysis we have above.

If $B \neq \emptyset$, then there exists $i \in \bar{\psi}^{*}(\mathbf{t})$ such that

$$
t_{i} \leq \bar{z}^{*}\left(\mathbf{t}_{-i}\right)<\bar{z}\left(\mathbf{t}_{-i}\right)=\sigma_{m}\left(\mathbf{t}_{-i}\right)
$$

where $m=m_{1}\left(\mathbf{t}_{-i}\right)$. By the definition of $m_{1}(\cdot)$ we have

$$
m \sigma_{m}\left(\mathbf{t}_{-i}\right) \leq 1 \text {. }
$$

By the single price property of $\overline{\mathcal{A}}^{*}$ we have

$$
\begin{aligned}
\left|\bar{\psi}^{*}(\mathbf{t})\right| & \leq\left|\left\{j: t_{j} \leq \bar{z}^{*}\left(\mathbf{t}_{-i}\right)\right\}\right| \\
& \leq\left|\left\{j: t_{j}<\bar{z}\left(\mathbf{t}_{-i}\right)\right\}\right| \\
& \leq m
\end{aligned}
$$

where (23) comes from the single price property and the other two inequalities are due to the analysis above. Again by the single price property we have

$$
\begin{aligned}
\sum_{j \in \bar{\psi}^{*}(\mathbf{t})} \bar{x}_{j}^{*}(\mathbf{t}) & =\left|\bar{\psi}^{*}(\mathbf{t})\right| \cdot \bar{z}^{*}\left(\mathbf{t}_{-i}\right) \\
& \leq m \sigma_{m}\left(\mathbf{t}_{-i}\right) \\
& \leq 1
\end{aligned}
$$

Thus $\overline{\mathcal{A}}^{*}$ is feasible.

From (9) we have

$$
1 \geq \bar{z}\left(\mathbf{t}_{-i}\right) \geq \bar{z}^{*}\left(\mathbf{t}_{-i}\right) \geq \sigma_{m}\left(\mathbf{t}_{-i}\right)-\left(\sigma_{m}\left(\mathbf{t}_{-i}\right)-\frac{1}{m+1}\right) \geq 0 .
$$

That is, $0 \leq \bar{z}^{*}\left(\mathbf{t}_{-i}\right) \leq 1$ for any $\mathbf{t}_{-i} \in T_{-i}$.

\section{Proof of Lemma 6}

Proof: For any t, let $m=|\mathcal{F}(\mathbf{t})|$, then

$$
m \sigma_{m}(\mathbf{t}) \leq 1
$$

and then

$$
(m-1) \sigma_{m}(\mathbf{t}) \leq 1
$$

so $|\overline{\mathcal{A}}(\mathbf{t})| \geq m-1=|\mathcal{F}(\mathbf{t})|-1$. Also,

$$
(m+1) \sigma_{m+2}(\mathbf{t}) \geq(m+1) \sigma_{m+1}(\mathbf{t})>1
$$

so $|\overline{\mathcal{A}}(\mathbf{t})| \leq|\mathcal{F}(\mathbf{t})|$.

\section{J. Proof of Lemma 7}

Proof: Just consider the case when all $n$ users bid $\frac{1}{m}$. Then $\mathcal{F}$ would admit $m$ out of the $n$ users randomly, while $\overline{\mathcal{A}}^{*}$ cannot admit any of the $n$ users. 


\section{K. Proof of Theorem 4}

Proof: Let $\mathbf{T}$ be the vector of random variables with values taken in $T$, and as usual let $\mathbf{T}_{i}$ and $\mathbf{T}_{-i}$ be the corresponding components. Note that $T \subset[0,1]^{n}$. Then the probability that $\overline{\mathcal{A}}^{*}$ and $\overline{\mathcal{A}}$ have different results is

$$
\begin{aligned}
D & =\operatorname{Pr}\left(\bar{z}^{*}\left(\mathbf{T}_{-i}\right)<\mathbf{T}_{i}<\bar{z}\left(\mathbf{T}_{-i}\right) \text { for some } i\right) \\
& \leq \sum_{i=1}^{n} \operatorname{Pr}\left(\bar{z}^{*}\left(\mathbf{T}_{-i}\right)<\mathbf{T}_{i}<\bar{z}\left(\mathbf{T}_{-i}\right)\right) \\
& =\sum_{i=1}^{n} \int_{B_{i}} f(\mathbf{t}) \mathrm{d} \mathbf{t} \\
& \leq \sum_{i=1}^{n} \mathcal{L}\left(B_{i}\right) K
\end{aligned}
$$

where

$$
B_{i}=\left\{\mathbf{t} \in T \mid \bar{z}^{*}\left(\mathbf{t}_{-i}\right)<t_{i}<\bar{z}\left(\mathbf{t}_{-i}\right)\right\}
$$

and $\mathcal{L}(\cdot)$ denotes the Lebesgue measure. Then

$$
\begin{aligned}
\mathcal{L}\left(B_{i}\right)= & \int_{B_{i}} 1 \mathrm{~d} \mathbf{t} \\
= & \int_{T_{-i}} \int_{\bar{z}^{*}\left(\mathbf{t}_{-i}\right)}^{\bar{z}\left(\mathbf{t}_{-i}\right)} 1 \mathrm{~d} t_{i} \mathrm{~d} \mathbf{t}_{-i} \\
= & \int_{\left\{\sigma_{1}\left(\mathbf{t}_{-i}\right)>\frac{1}{2}\right\}} d_{1}\left(\sigma_{1}\left(\mathbf{t}_{-i}\right)-\frac{1}{2}\right) \mathrm{d} \mathbf{t}_{-i} \\
& +\int_{\left\{\frac{1}{2} \geq \sigma_{2}\left(\mathbf{t}_{-i}\right)>\frac{1}{3}\right\}} d_{2}\left(\sigma_{2}\left(\mathbf{t}_{-i}\right)-\frac{1}{3}\right) \mathrm{d} \mathbf{t}_{-i} \\
& +\ldots \\
& +\int_{\left\{\frac{1}{n-1} \geq \sigma_{2}\left(\mathbf{t}_{-i}\right)>\frac{1}{n}\right\}} d_{n-1}\left(\sigma_{n-1}\left(\mathbf{t}_{-i}\right)-\frac{1}{n}\right) \mathrm{d} \mathbf{t}_{-i} \\
\leq & \frac{1}{2} d_{1}+\left(\frac{2}{3}-\frac{1}{2}\right) d_{2}+\cdots+\left(\frac{n-1}{n}-\frac{n-2}{n-1}\right) d_{n-1} \\
\leq & \max _{1 \leq j \leq n-1} d_{j}\left(1-\frac{1}{n}\right) \\
\leq & \max _{1 \leq j \leq n-1} d_{j} \cdot
\end{aligned}
$$

Thus

$$
D \leq d n K,
$$

where $d=\max _{1 \leq j \leq n-1} d_{j}$. 